\title{
Radiologically Undetectable Latent Pulmonary Lymphocyte Infiltration Suggesting the Need for Early Glucocorticoid Therapy: An Autopsy Case of Rapidly Progressive COVID-19 Pneumonia with Lymphopenia
}

Naoki Tanomogi ${ }^{1}$, Toshinori Otani ${ }^{2}$, Junko Araki ${ }^{3}$, Ayumi Sugitani ${ }^{2}$, Shingo Itagaki ${ }^{2}$, Takahiro Kiriu ${ }^{2}$ and Kota Shimada ${ }^{1}$

\begin{abstract}
:
We herein report a fatal case of coronavirus disease 2019 (COVID-19) pneumonia with rapid progression of respiratory failure and lymphopenia. Excessive recruitment and sequestration of lymphocytes in the lung were suggested as the pathophysiology underlying COVID-19-associated lymphopenia. Interestingly, the autopsy in this case revealed lymphocytic infiltration in the lungs even at sites that appeared normal on autopsy imaging. These findings suggest that in COVID-19 cases with risk factors of severe exacerbation, early glucocorticoid administration should be considered, especially if lymphopenia is present, even if the imaging findings show only mild abnormalities.
\end{abstract}

Key words: COVID-19 pneumonia, coronavirus disease 2019, SARS-CoV-2, autopsy, lymphocytic infiltration, lymphopenia

(Intern Med 60: 2145-2148, 2021)

(DOI: 10.2169/internalmedicine.7234-21)

\section{Introduction}

Severe acute respiratory dysfunction often occurs in patients with coronavirus disease 2019 (COVID-19). Glucocorticoid therapy is indicated for the treatment of COVID19 (1), but there is no clear consensus as to when to initiate it. We herein report a fatal case of COVID-19 with rapid progression of respiratory failure. An autopsy provided pathological and radiological findings supporting the efficacy of early glucocorticoid administration for COVID-19.

\section{Case Report}

An 85-year-old man with chronic kidney disease and schizophrenia was transferred to our hospital with a suspected severe acute respiratory syndrome coronavirus 2 (SARS-CoV-2) infection. He had been admitted to the previous hospital for schizophrenia during an outbreak of
COVID-19 in his ward. On presentation, he had a two-day history of cough and a fever (day 1), and a positive reverse transcription-polymerase chain reaction test confirmed COVID-19.

A physical examination revealed blood pressure 162/90 $\mathrm{mmHg}$, heart rate $83 / \mathrm{min}$, temperature $38.8^{\circ} \mathrm{C}$, respiratory rate $20 / \mathrm{min}$, and oxygen saturation $97 \%$ on room air. Laboratory tests at admission showed leukopenia but no increase in serum $\mathrm{C}$-reactive protein (CRP) levels. Chest X-ray showed slight, bilateral ground-glass lesions (Fig. 1A). The fever persisted after the transfer, and the serum CRP level increased. On hospitalization day 6, his $\mathrm{SpO}_{2}$ decreased, and oxygen administration $(1 \mathrm{~L} / \mathrm{min})$ was started. On hospitalization day 8 , his oxygen demand increased to $2 \mathrm{~L} / \mathrm{min}$. Chest $\mathrm{X}$-ray showed exacerbation of the ground-glass opacity (Fig. 1B). Dexamethasone was not used to treat COVID-19 due to the lack of any established recommendations at the time (2).

On the evening of hospitalization day 9 , oxygen demand

${ }^{1}$ Department of Rheumatic Diseases, Tokyo Metropolitan Tama Medical Center, Japan, ${ }^{2}$ Department of Pathology, Tokyo Metropolitan Tama Medical Center, Japan and ${ }^{3}$ Department of Radiology, Tokyo Metropolitan Tama Medical Center, Japan Received: February 2, 2021; Accepted: March 29, 2021; Advance Publication by J-STAGE: May 14, 2021 Correspondence to Dr. Naoki Tanomogi, tanomoginaokiwind@yahoo.co.jp 


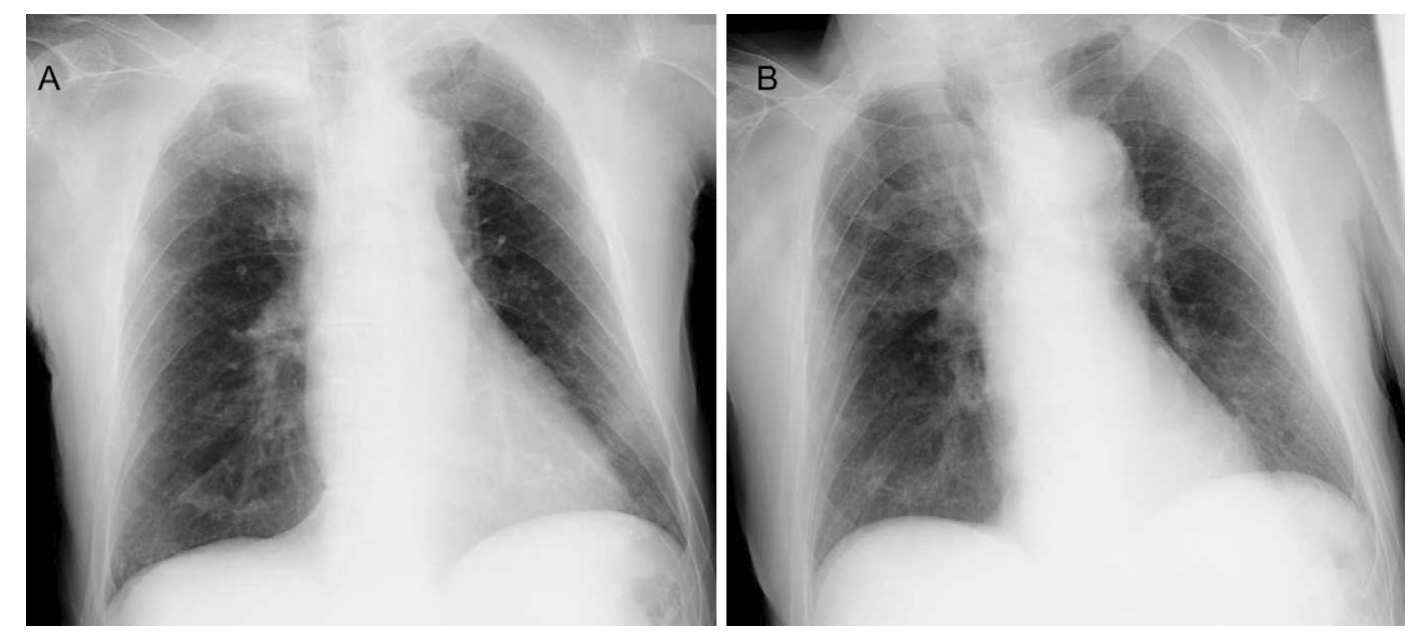

Figure 1. Chest X-ray on hospitalization day 1 (A) and 8 (B). Slight, bilateral ground-glass opacity was evident on hospitalization day 1 and showed exacerbation on day 8.

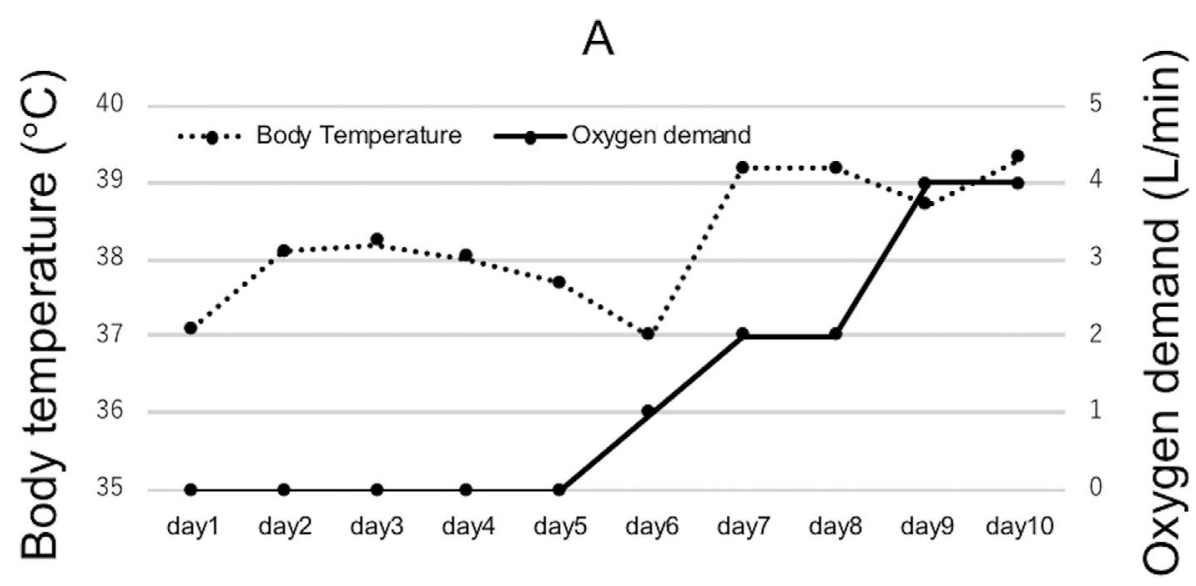

B

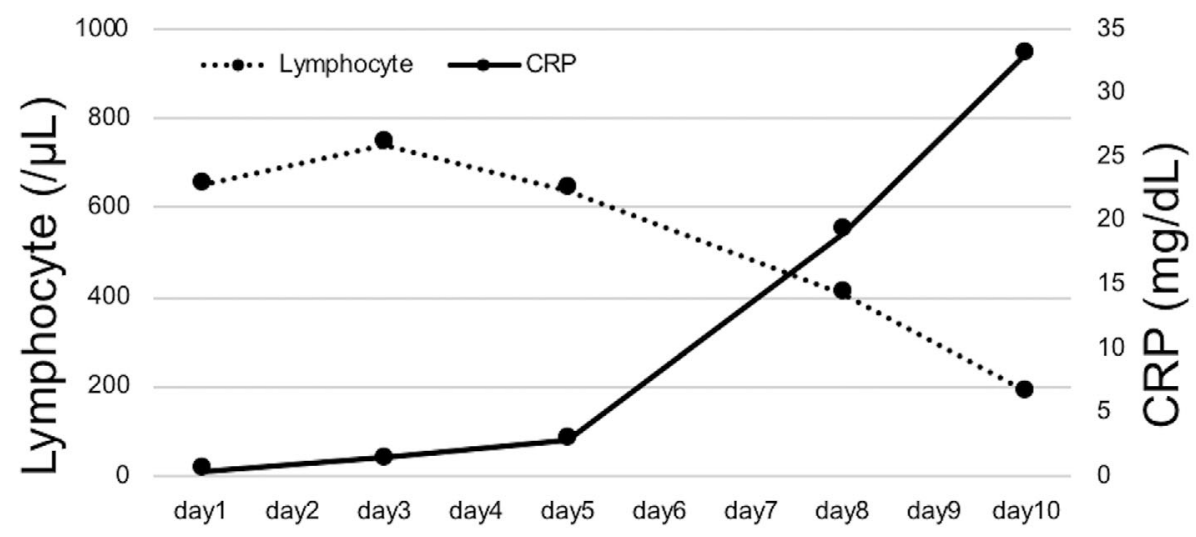

Figure 2. Lymphocytes decreased inversely to the exacerbation of the body temperature, hypoxemia, and CRP. The course of the body temperature and oxygen demand (A). The course of lymphocyte count and CRP levels (B).

rapidly increased from 2 to $4 \mathrm{~L} / \mathrm{min}$. Given the grave condition of the patient, after careful discussion with the patient's family, the decision was made not to resuscitate. From 6 am on hospitalization day 10 , tachypnea with a respiratory rate of 30 to 40 breaths per minute appeared. At 7:40, his pulse suddenly dropped from 105 beats per minute to cardiac ar- rest. Only four days had lapsed from the commencement of oxygen administration to death (Fig. 2A). Post-mortem computed tomography (CT) performed four hours after death showed extensive ground-glass opacities in the bilateral lungs that were more severe in the lower dorsal lobes than in the upper lobes (Fig. 3), as the upper lobes of the lungs 


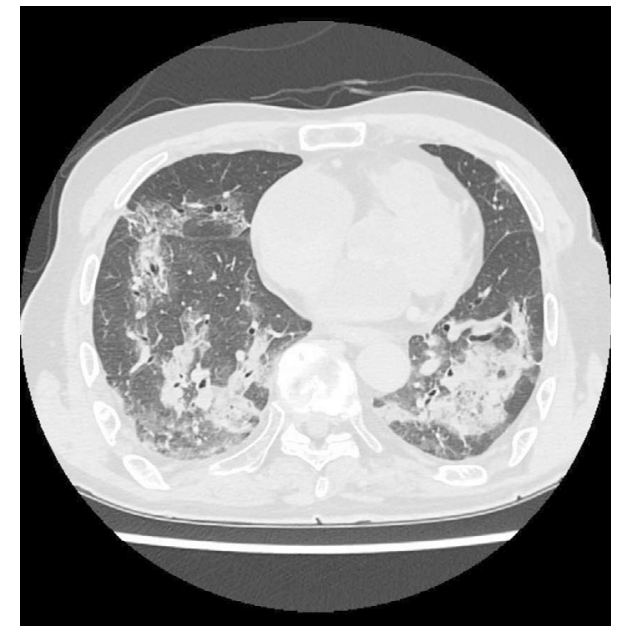

Figure 3. Postmortem CT findings showing extensive bilateral ground-glass opacities in the lower dorsal region.

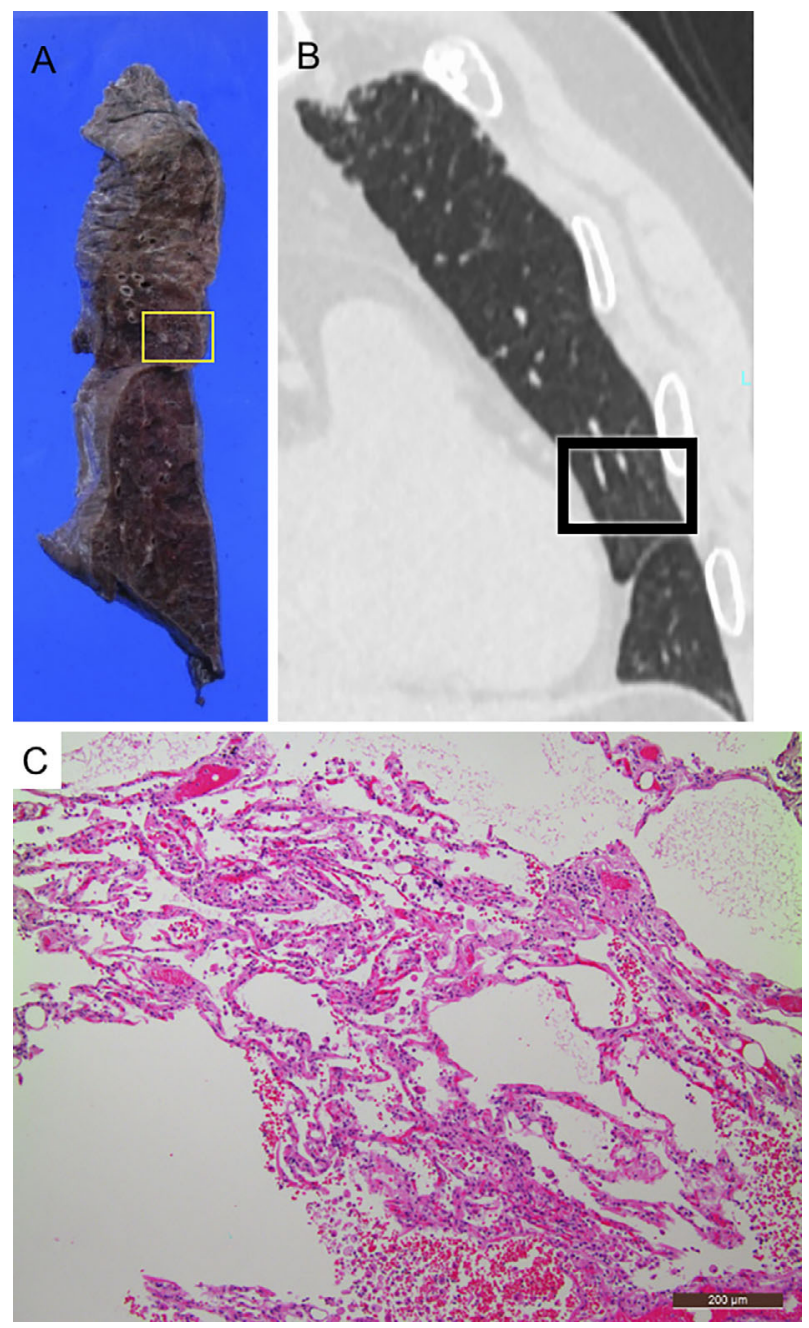

Figure 5. Lymphocyte infiltlation confirmed in areas without abnormal findings on CT. Macroscopic findings (A), CT autopsy findings (B), and microscopic findings (C). The area shown in (C) corresponds to the area in the yellow (A) and black (B) boxes.

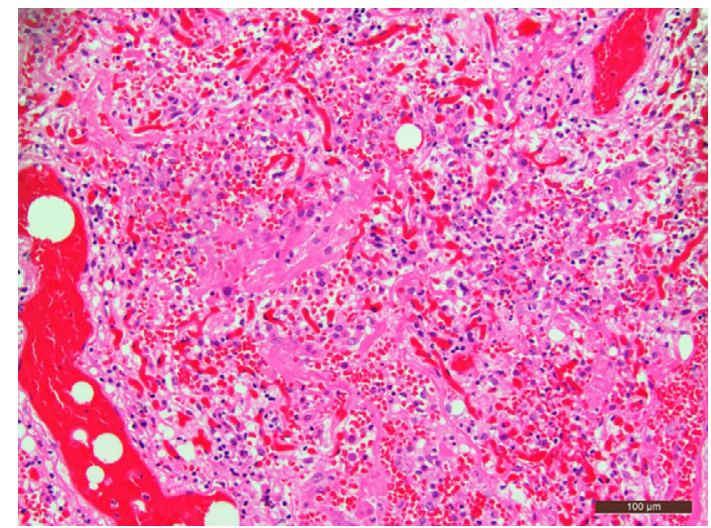

Figure 4. Pathological findings of the dorsal side of the lower lung lobe showing significant alveolar hemorrhaging.

appeared relatively normal.

Because the patient's death had followed a relatively rapid clinical course, a pathological autopsy was performed to inquire into the direct cause of death. The autopsy of the body (except for the brain) was conducted 22 hours after death. No pulmonary embolism or myocardial infarction was observed on a macroscopic examination. Congestion was systemically prominent. Petechiae were scattered on the lung surface. Microscopically, findings corresponding to the exudative phase of diffuse alveolar damage (DAD), including hyaline membrane formation, interstitial edema, lymphocyte infiltration, and type I alveolar epithelial cell shedding, were diffusely observed in the lungs. Significant alveolar hemorrhaging had occurred at the dorsal side of the lower lobe of the bilateral lungs (Fig. 4). Lymphocyte infiltration in the periphery of the blood vessels and edematous thickening of the blood vessel wall were observed. Lymphocyte infiltlation into the interstitial tissue was also confirmed in areas where there were no abnormal findings on CT (Fig. 5). Based on the absence of pulmonary embolism and myocardial infarction, COVID-19 pneumonia was anatomically diagnosed as the cause of death.

\section{Discussion}

Anti-inflammatory therapies are considered effective for the treatment of COVID-19 pneumonia. There are several clinical trials in which glucocorticoid therapy has shown good efficacy. In one randomized, open-label, clinical trial in Brazil enrolling 299 patients with confirmed or suspected COVID-19 who were receiving mechanical ventilation, intravenous dexamethasone plus standard care resulted in a statistically significant increase in the number of ventilatorfree days compared with standard care alone (3). In another randomized open-label trial in the United Kingdom, oral or intravenous dexamethasone reduced the 28-day mortality among hospitalized patients to a greater extent than usual care alone (4). In that trial, which included hospitalized patients with COVID-19, 2,104 and 4,321 patients were randomized to a dexamethasone treatment group and a usual 
care group, respectively. As a result, 482 patients $(22.9 \%)$ in the dexamethasone group and 1,110 patients $(25.7 \%)$ in the usual care group died within 28 days after randomization [age-adjusted rate ratio, 0.83; 95\% confidence interval $(\mathrm{CI})$, 0.75 to $0.93 ; \mathrm{P}<0.001]$ (4). In contrast, in a randomized, double-blind, sequential trial conducted in France that included 149 critically ill patients with biologically confirmed or suspected COVID-19 (based on chest CT findings in the absence of any other cause of pneumonia), low-dose hydrocortisone did not significantly reduce treatment failure (defined as death or persistent respiratory support) at day 21 compared with a placebo (5). Furthermore, the incidence of adverse events can increase even with short-term glucocorticoid administration (6). However, in a meta-analysis of 7 trials enrolling 1,703 critically ill patients with COVID-19, glucocorticoid therapy reduced mortality to a greater extent than standard care or a placebo (1). Based on these somewhat contradictory findings, it has been suggested that oxygen demand, severity of lung imaging findings, and risk factors, such as age and complications, should be considered when weighing the merits of using glucocorticoid therapy.

Lymphopenia is another risk factor of COVID-19 pneumonia aggravation (7). One hypothesis of the pathophysiology underlying COVID-19-associated lymphopenia is excessive recruitment and sequestration of lymphocytes in the lung (8). Our patient had lymphocyte infiltlation in the interstitial tissue of the lungs, and his lymphopenia progressed as the disease progressed (Fig. 2B), supporting this hypothesis. Furthermore, lymphocyte infiltlation was confirmed at sites that appeared normal on CT. Given the discrepancy between the imaging and histopathological findings, lymphocyte infiltlation may have existed in a wider area than was indicated by radiographic findings.

Generally, glucocorticoids induce apoptosis of lymphoid cells (9); lymphocyte infiltlation in the interstitial tissue in COVID-19 is thus the target of glucocorticoid therapy (10). In the present case, the DAD findings were the main histopathological change, and alveolar hemorrhaging was also observed. Therefore, it might have been difficult to save this patient even if glucocorticoid therapy had been started soon after the development of adult respiratory distress syndrome (ARDS). However, since the lymphopenia in this case preceded the rapid progression of the pulmonary lesions, early glucocorticoid administration might have conversely led to a better clinical course. Thus, in patients with a risk of severe exacerbation, lymphopenia can be a factor justifying early glucocorticoid administration, even if imaging studies reveal only mild abnormalities.

Consent to publish the details of the case was obtained from the family of the patient.

The authors state that they have no Conflict of Interest (COI).

\section{Acknowledgement}

We thank Dr James R. Valera for his assistance in editing the manuscript.

\section{References}

1. Sterne JAC, Murthy S, Diaz JV, et al.; WHO Rapid Evidence Appraisal for COVID-19 Therapies (REACT) Working Group. Association between administration of systemic corticosteroids and mortality among critically Ill patients with COVID-19: a metaanalysis. JAMA 324: 1330-1341, 2020.

2. Tang C, Wang Y, Lu H, Guan Z, Gu J. Caution against corticosteroid-based COVID-19 treatment. Lancet 395: 1759-1760, 2020.

3. Tomazini BM, Maia IS, Cavalcanti AB, et al. Effect of dexamethasone on days alive and ventilator-free in patients with moderate or severe acute respiratory distress syndrome and COVID-19: the CoDEX randomized clinical trial. JAMA 324: 1308, 2020.

4. Horby P, Lim WS, Emberson JR, et al.; Recovery Collaborative Group. Dexamethasone in hospitalized patients with Covid-19 preliminary report. NEJM 384: 693-704, 2021.

5. Dequin PF, Heming N, Meziani F, et al. Effect of hydrocortisone on 21-day mortality or respiratory support among critically Ill patients with COVID-19. JAMA 324: 1-9, 2020.

6. Waljee AK, Rogers MA, Lin P, et al. Short term use of oral corticosteroids and related harms among adults in the United States: population based cohort study. BMJ 357: 1415, 2017.

7. Tan L, Wang Q, Zhang D, et al. Lymphopenia predicts disease severity of COVID-19: a descriptive and predictive study. Signal Transduct Target Ther 5: 33, 2020.

8. Torres Acosta MA, Singer BD. Pathogenesis of COVID-19induced ARDS: implications for an ageing population. Eur Respir J 56: 2002049, 2020.

9. Schwartzman RA, Cidlowski JA. Glucocorticoid-induced apoptosis of lymphoid cells. Int Arch Allergy Immunol 105: 347-354, 1994.

10. Singh AK, Majumdar S, Singh R, Misra A. Role of corticosteroid in the management of COVID-19: a systemic review and a Clinician's perspective. Diabetes Metab Syndr 14: 971-978, 2020.

The Internal Medicine is an Open Access journal distributed under the Creative Commons Attribution-NonCommercial-NoDerivatives 4.0 International License. To view the details of this license, please visit (https://creativecommons.org/licenses/ by-nc-nd/4.0/).

\section{(C) 2021 The Japanese Society of Internal Medicine} Intern Med 60: 2145-2148, 2021 